\title{
Investigation on high inductive helical supported magnetically insulated transmission line on a 10-stage linear transformer driver system
}

\author{
Wenkang Zou, ${ }_{1}$ Lin Chen, ${ }^{1}$ Dagang Liu, ${ }^{2}$ Le Zhang, ${ }_{1}^{1}$ Laqun Liu, ${ }^{2}$ Liangji Zhou, ${ }^{1}$ Meng Wang, ${ }^{1}$ Bing Wei, ${ }^{1}$ Fan Guo, ${ }^{1}$ \\ Xueqiong Wang, ${ }^{2}$ Yingmin Dai, ${ }^{1}$ Weiping Xie, ${ }^{1}$ and Jianjun Deng ${ }^{1}$ \\ ${ }^{1}$ Institute of Fluid Physics, CAEP, P.O. Box 919-108, Mianyang 621900, China \\ ${ }^{2}$ School of Physical Electronics, University of Electronic Science and Technology of China, Chengdu 610054, China
}

(Received 19 October 2011; published 12 November 2012)

\begin{abstract}
Magnetically insulated transmission lines (MITLs), which could transfer power density up to TW/ $\mathrm{cm}^{2}$, are one of the most important technologies in pulsed power. In pulsed power systems for the $Z$-pinch fusion or radiography, a long MITL acts as a transmission line as well as a spatial isolation between load and driver. The length of MITLs in such systems will be up to a few, even tens of meters. However, the anode and cathode (A-K) gap is only a few centimeters to make the centering of the MITL's electrodes be one of the most challenging issues. Cathodes of long coaxial MITLs, such as that of Hermes-III and RITS, are fixed at the low voltage end while keeping the other end free of support. However, such a method will be very difficult for longer MITLs due to gravity and engineering reasons. An interesting question for such MITL design is to find a way to position the electrodes to the ideal position while hardly damaging the power flow. It is also a very practical concern in the construction of large pulsed-power facilities. In this paper, a high inductive helical supported MITL in a 10-stage linear transformer driver system is investigated. Both experiments and particle-in-cell simulations show that magnetic insulation is well established and power flow could be transmitted to load efficiently.
\end{abstract}

PACS numbers: 52.58.Lq, 84.70.+p, 84.60.Ve

\section{INTRODUCTION}

In large scale pulse drivers, loads are usually placed several meters away from the primary energy storage. A magnetically insulated transmission line (MITL) provides an efficient way to transmit high power pulse (power density up to $\mathrm{TW} / \mathrm{cm}^{2}$ or current density up to $\mathrm{MA} / \mathrm{cm}$ ) to load, making it an essential and indispensable component for large pulsed power drivers [1].

Two typical MITLs in structure are applied in pulse drivers in the literature. In low impedance drivers, such as the $Z$ machine [2], the Primary Test Stand (PTS) [3], and future petawatt-class driver [4], the MITL usually works as the joint part of identical modules. Power flow from individual modules is converged at the MITL and then transmitted to load. The MITL in such drivers usually has concentric structure. While in high impedance facilities which usually drive particle beam diodes, such as Hermes-III [5], RITS [6], and the linear transformer driver (LTD) system in this paper, the MITL usually has coaxial structure. In these facilities, the inner electrode of the MITL is fixed at the ground potential end, and the other end, where the particle beam is generated, is usually free of support. A principal engineering consideration in such a design is the misalignment of the inner electrode.

Published by the American Physical Society under the terms of the Creative Commons Attribution 3.0 License. Further distribution of this work must maintain attribution to the author(s) and the published article's title, journal citation, and DOI.
Investigation on the Hermes-III showed a misalignment of $5 \%$ of the A-K gap produced no measurable loss, while noticeable loss occurred when the gap was misaligned by $25 \%$ [7]. As a deflection of several centimeters would emerge due to the gravity for a cantilevered metallic line with tens of meters in length, the MITL cathode of Hermes-III was divided into sections and a complicated set of adjusting procedure was employed to control the alignment error within criteria.

With the development of fast LTD [8,9] in the past decade, new $\mathrm{x}$-ray flash radiography facilities with a voltage of several megavolts are turning into reality from concept [10]. LTD is modular, flexible, and compact compared with traditional pulse forming technologies. However, the output voltage of a single stage is only about $100 \mathrm{kV}$. To obtain high voltage, many stages need to be connected in series. For the same voltage, the length of voltage adder with LTD might be not less than that of Hermes-III or RTIS. Therefore, controlling the electrode misalignment within physical allowed criteria will be much more challenging.

For LTD based Z-pinch drivers, many LTD modules (each has tens of stages connected in series with MITL or water line) are connected together at the high voltage end and then drive a common load. There are at least three questions that need to be considered in such a situation. The first one is to choose a good transition in structure from coax into triplate, then into conical biplate to accommodate with load. The second one is to support the target section into its ideal position in vacuum, where the magnetic 
insulation should be preserved. The third one is about the entry of premagnetize current for cores, by which the magnetic field is reset to negative maximum to ensure no saturation would happen during the pulse. But during normal discharge, the premagnetizing loop should break or work as a high impedance passage.

Motivated by these questions, we investigate the possibility of supporting MITLs with high inductive helical on a 10 -stage LTD system. The paper is arranged as follows. Section II describes the design and performance of the LTD system. Section III is devoted to the experiment results with and without high inductive helical support. Section IV presents three-dimensional (3D) particle-in-cell (PIC) simulation results to benchmark the experiments. The summary and conclusions are presented in Sec. V.

\section{DESIGN AND PERFORMANCE OF THE LTD SYSTEM}

A 10-stage LTD system was developed to drive the e-beam diode in 2010 at China Academy of Engineering Physics (CAEP). It is the first $\sim 100$ ns LTD system in China.

\section{A. Descriptions and test results of the LTD stages}

The LTD stages finally assembled in the system have a similar design with the prototype (see Fig. 1) in Ref. [11]. Twenty $100 \mathrm{kV} / 20 \mathrm{nF}$ capacitors (Model CBB93-1000.02, by Wuxi City Ximai Electric Components Co.) and ten multigap switches (MGS) form ten bricks. Each brick contains two series capacitors and one MGS. The high voltage terminals of the capacitor are located at opposite sides of the body to make it more compact and convenient to assemble into the LTD stage. Several MGS models had been developed since 2005. The final model (see Fig. 2) used in the system has six gaps. Each gap has a length of $5 \mathrm{~mm}$. Corona needles are used for voltage distribution between gaps. Polymethyl methacrylate is used as the body

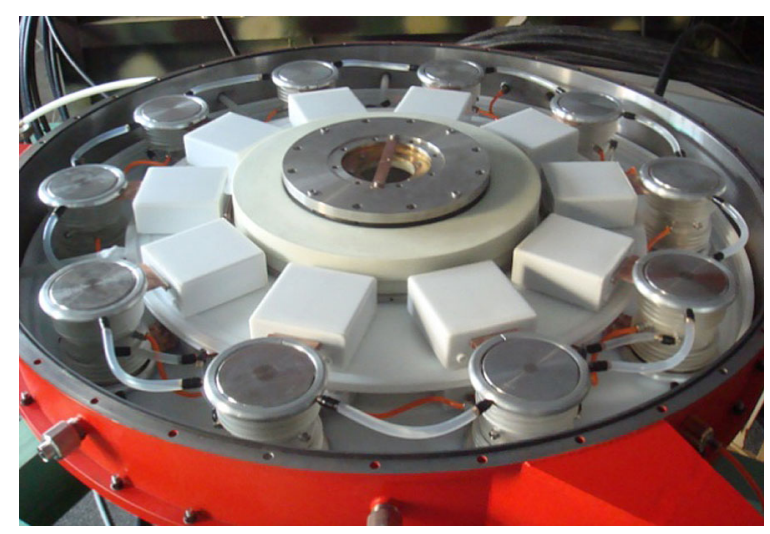

FIG. 1. Prototype of the $100 \mathrm{kA}$ LTD stage. There are ten bricks in parallel. Each brick has two $20 \mathrm{nF}$ capacitors and one MGS in series. The maximum energy storage of a LTD stage is $2 \mathrm{~kJ}$ when capacitors are all charged to $100 \mathrm{kV}$.

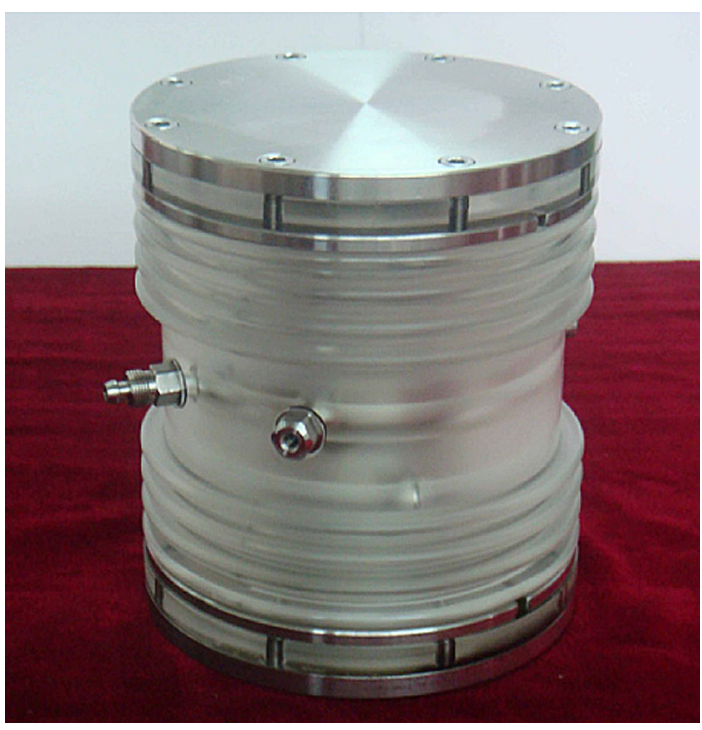

FIG. 2. The final MGS model used in the LTD system. The model has six gaps with total length of $30 \mathrm{~mm}$. The maximum working voltage is about $200 \mathrm{kV}$ and the average jitter is about $2.5 \mathrm{~ns}$.

material of the switch shell. A mixture of $\mathrm{SF}_{6}$ and nitrogen with a pressure of 1.3 ata is filled into the switch chamber as insulation media. The maximum working voltage for the MGS is about $200 \mathrm{kV}$ and the average jitter is about $2.5 \mathrm{~ns}$ at the working ratio (applied voltage to its self-breakdown voltage) of about $\sim 70 \%$.

Magnetic cores, which were manufactured by Sichuan Liyuan Electric Inc., Ltd, are employed to reduce the parasitic current to cavity shell. Each LTD stage has six circular magnetic cores, which are wound by $25 \mu \mathrm{m} \times 20 \mathrm{~mm}$ amorphous iron tapes. Polyester films are used as insulation between adjacent tapes. In each LTD stage, the effective cross section of the core is about $100 \mathrm{~cm}^{2}$. The diameter of the LTD stage is $1.5 \mathrm{~m}$, and the height is $20.5 \mathrm{~cm}$.

All ten LTD stages were tested separately with water solution resistors, which were placed in the middle of the stages. The voltage and current of the resistors were recorded. Table I presents test results for all ten stages when the charging voltage is $95 \mathrm{kV}$. The results show that load currents are around $100 \mathrm{kA}$ and voltages are all above $110 \mathrm{kV}$. In the table, the fifth stage gives the lowest current, because the highest resistance $(1.64 \Omega)$ was used.

When the LTD stages were tested, the trigger voltage had a rise time of $48 \mathrm{~ns}$ from zero to $110 \mathrm{kV}$. However, after all LTD stage tests were finished, the same trigger was improved and then applied to the LTD system. Its rise time reduced to 38 ns. Figure 3 shows a typical voltage waveform of the improved trigger. Its FWHM is about $110 \mathrm{~ns}$. The trigger has ten output cables with the same length to make all the LTD stages be triggered simultaneously. Fitting from experimental data, the equivalent capacitance, inductance, and resistance of a LTD stage are $100 \mathrm{nF}$, $30 \mathrm{nH}$, and $0.14 \Omega$, respectively. 
TABLE I. Test results for all ten LTD stages when the charging voltage $\left(U_{C}\right)$ is $95 \mathrm{kV}$. Voltage $U_{L}$ and current $I_{L}$ are recorded on a water solution resistor. In these tests, the resistance varies from 1 to $2 \Omega$. Delay $\tau_{d}$ is defined as the time duration from the start of the trigger pulse to the start of current pulse. Current rise time $\tau_{r}$ is the time duration from zero-to-maximum current, and $\tau_{\text {FWHM }}$ the full width at half maximum.

\begin{tabular}{lccrccc}
\hline \hline LTD stage & $U_{C}(\mathrm{kV})$ & $U_{L}(\mathrm{kV})$ & $I_{L}(\mathrm{kA})$ & $\tau_{d}(\mathrm{~ns})$ & $\tau_{r}(\mathrm{~ns})$ & $\tau_{\mathrm{FWHM}}(\mathrm{ns})$ \\
\hline 1 & 95 & 111.0 & 98.8 & 56 & 82 & 143 \\
2 & 95 & 119.5 & 102.9 & 59 & 84 & 148 \\
3 & 95 & 128.0 & 110.0 & 54 & 91 & 160 \\
4 & 95 & 129.6 & 109.7 & 61 & 93 & 148 \\
5 & 95 & 149.5 & 90.9 & 57 & 86 & 150 \\
6 & 95 & 142.7 & 116.0 & 60 & 88 & 145 \\
7 & 95 & 140.8 & 106.0 & 59 & 89 & 150 \\
8 & 95 & 142.1 & 103.6 & 55 & 90 & 144 \\
9 & 95 & 135.3 & 103.4 & 59 & 88 & 152 \\
10 & 95 & 139.8 & 101.4 & 63 & 89 & 147 \\
\hline \hline
\end{tabular}

\section{B. General description of the LTD system}

As shown in Fig. 4, ten LTD stages are mounted vertically on a supporting structure. The structure is situated on rails and could be moved back and forth to facilitate different load arrangement. The LTD stages are connected one by one and are compressed inwards from two sides of the supporting structure. A cylindrical hollow stalk penetrates all LTD cavities from their centers. The stalk is fixed to a flange at the left side of the first LTD cavity. The stalk and output electrodes of all LTDs form the coaxial vacuum MITL.

The system has two hundred capacitors and one hundred MGS in all. The maximum energy storage is $20 \mathrm{~kJ}$ when capacitors are charged to $100 \mathrm{kV}$. But allowing for reliability and stability, the maximum charging voltage is $95 \mathrm{kV}$

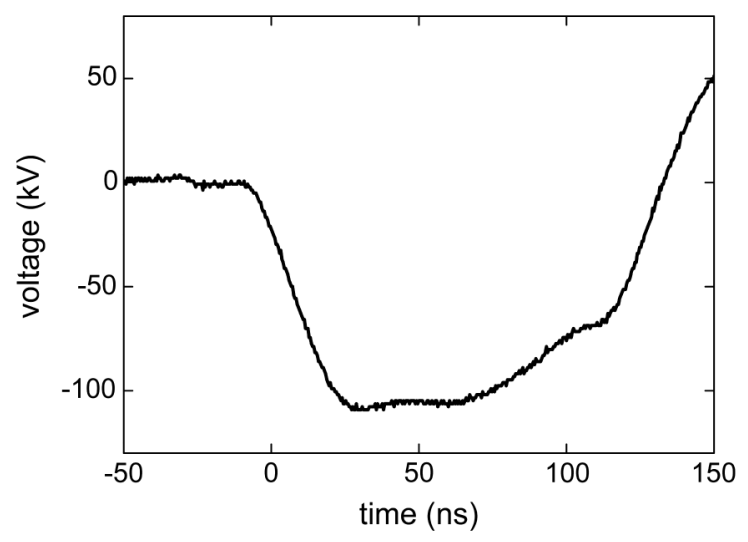

FIG. 3. Typical voltage waveform of the improved trigger generator. The maximum voltage is $110 \mathrm{kV}$ with zero-tomaximum rise time of $38 \mathrm{~ns}$, and a FWHM of $110 \mathrm{~ns}$. The trigger generator has ten output cables with the same length. Each cable can trigger all ten MGS in a LTD stage. All ten LTD stages in the system are triggered simultaneously.

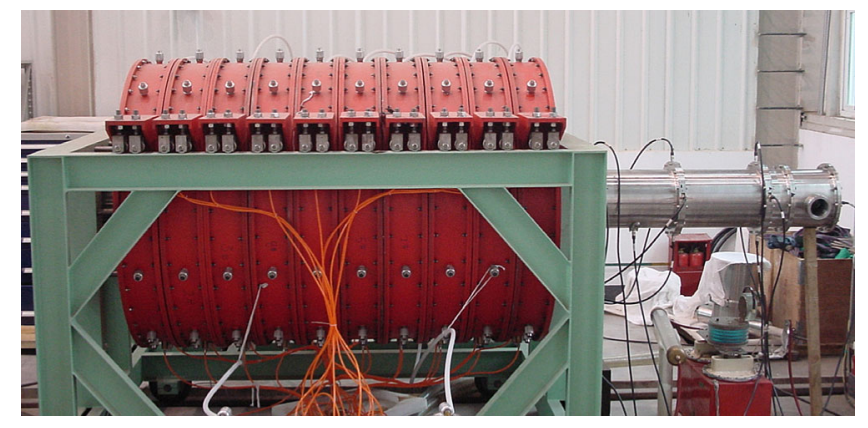

FIG. 4. Photo of the 10-stage LTD system, in which ten identical $100 \mathrm{kA}$ LTD stages are connected in series. Supporting structure of the system is situated on rails and is able to be moved back and forth. In this photo, a straight extensional MITL without inductive support is added to output end.

in our experiments. Typically, when charging to $90 \mathrm{kV}$, more than $100 \mathrm{kA}$ current could be obtained on a $10 \Omega$ resistive load. When the charging voltage is below $90 \mathrm{kV}$ and the shot interval is above two minutes, self-breakdown probability of the system is less than $5 \%$.

The system works in negative polarity so far. The output electrodes of the LTDs, whose diameters are $30 \mathrm{~cm}$, form the MITL anode, and the inner electrode is the MITL cathode. Both taper and straight cathode structures, which are made of aluminum alloy $2 \mathrm{~A} 12$, were tested in different experimental states. For the taper one, the diameter reduces from 27 to $22 \mathrm{~cm}$. Correspondingly, the vacuum impedance increases from 6.3 to $18.6 \Omega$. For the straight cathode, the diameter is $24 \mathrm{~cm}$, and the line has a constant vacuum impedance of $13.4 \Omega$. The length of cathode stalks in the LTD part is about $2.3 \mathrm{~m}$. Different extensional MITLs are installed between the end of the stalk and the load to conduct experiments with and without helical support.

\section{DESIGN AND EXPERIMENT OF EXTENSIONAL MITL WITH AND WITHOUT HIGH INDUCTIVE HELICAL SUPPORT}

To evaluate the influence of helical support to magnetic insulation, a MITL of $1.2 \mathrm{~m}$ was designed and connected to the output of the LTD system. Comparison experiments with and without the high inductive support at the extensional MITL were conducted.

\section{A. Design of the high inductive helical support}

In the previous experiment setup, the MITL cathode was fixed at the ground potential end and the high voltage end was free of support. Adjusting screws in a circular arrangement at one side of the supporting structure were used to center the cathode. To verify the proposed idea of this paper, a cylindrical stainless tube in which a helical inductor links the cathode and anode was designed at the extensional section of the MITL. The helical inductor in 
the tube should work as a cathode support and premagnetizing current entry to all magnetic cores simultaneously. Characteristic frequency of the LTD circuit, load impedance, and the current shunting from the support were considered when the structural parameters of the helical were determined.

According to circuit analysis, current rise time of the system is less than $100 \mathrm{~ns}$ and the dominant frequency of current is about $2.5 \mathrm{MHz}$. As the impedance of an inductor is proportional to the pulse frequency applied to it, when the magnetic core is premagnetized, one feasible solution is to apply a much slower and opposite current pulse (with rise time of a few microseconds) to the helical. Then, the inductor could be treated as a short circuit.

Since the helical inductor is paralleled to the diode, another factor in designing is to decide how much current will be allowed to shunt across it. In our design, loss of $10 \%$ in total current is the upper limit. For the LTD system, reasonable load impedance is 5-10 $\Omega$. Therefore, an equivalent impedance of $100 \Omega$ for the support is sufficient. Accordingly, the required inductance of the helical support is more than $6 \mu \mathrm{H}$.

A schematic diagram and parameter definition for the helical inductor are shown in Fig. 5. For such an inductor, Ref. [12] gives

$$
L=\frac{\mu_{0}}{4 \pi} W^{2} d \phi
$$

where $L$ is the inductance, $\mu_{0}$ is the permeability in vacuum, $W$ is the windings, $d$ is the diameter, $a$ is the length, and $\phi$ is a function of $a / d$, which could be obtained from numerical table in Ref. [12].

As the real supporting structure is composed not only by the helical inductor, but also a returning cavity, thus the effective inductance of the supporting structure will be lower than that of Eq. (1).

Empirically, when the needed inductance and corresponding dimensional parameters are determined, $W$ and $a$ could be increased to get larger nominal inductance. Here we choose $L=10 \mu \mathrm{H}$ (margin was reserved with
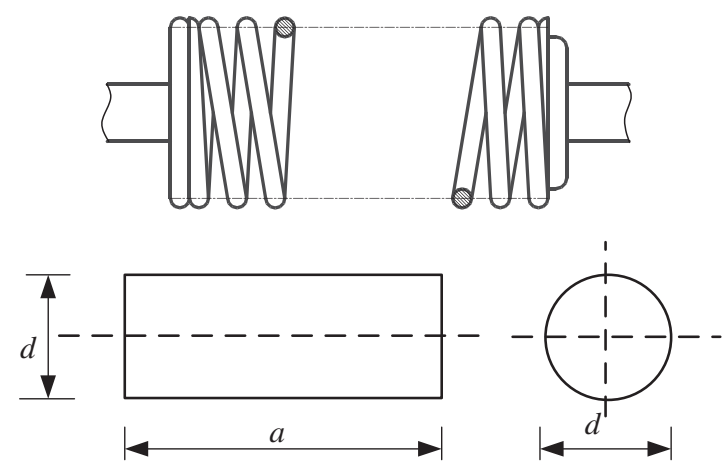

FIG. 5. A schematic diagram of helical inductor with windings $W$, length $a$, and diameter $d$. respect to $6 \mu \mathrm{H}$ ) for $a=d=10 \mathrm{~cm}$. Correspondingly, $\phi$ equals 6.8 , then $W=12$ is determined. While actually, $W=19.5$ and $a=30 \mathrm{~cm}$ are used in our design to give a larger margin. Stainless steel wire with a diameter of $8 \mathrm{~mm}$ is used to wind the inductor. The distance between adjacent windings is also $8 \mathrm{~mm}$.

Figure 6 illustrates the structure design and probe layout of the helical support. Helical inductor (5) is installed vertically in a cylindrical tube, whose inner diameter is $24.5 \mathrm{~cm}$. The tube, whose total height is about $730 \mathrm{~mm}$, is divided into three parts in the vertical direction. The bottom part is a tee joint. Upstream and downstream of the MITL are connected to the two horizontal ports. The middle part of the tube is used to facilitate fixing operation. The top part is to enclose the vacuum chamber.

A nylon insulation plate (3) is used to radially position the inductor. A copper rod (4), which penetrates the plate and connects with upper end of inductor, serves as entry for premagnetizing current. A corrugated pipe (2) breaks the inductor with the anode when applying premagnetizing current to the system. When the LTD system discharges, the corrugated pipe connects the inductor and anode again. When all parts of the helical support are in their right position, the measured effective inductance is $9.7 \mu \mathrm{H}$.

In coaxial MITLs, the electromagnetic wave propagates in TEM mode. As the helical inductor is implemented, field distribution will be disturbed, and parts of the electron will be launched and might drift into the vertical tube where the inductor exists. To prevent this trend and reduce the field enhancement, a grading structure (6) is used. The PIC

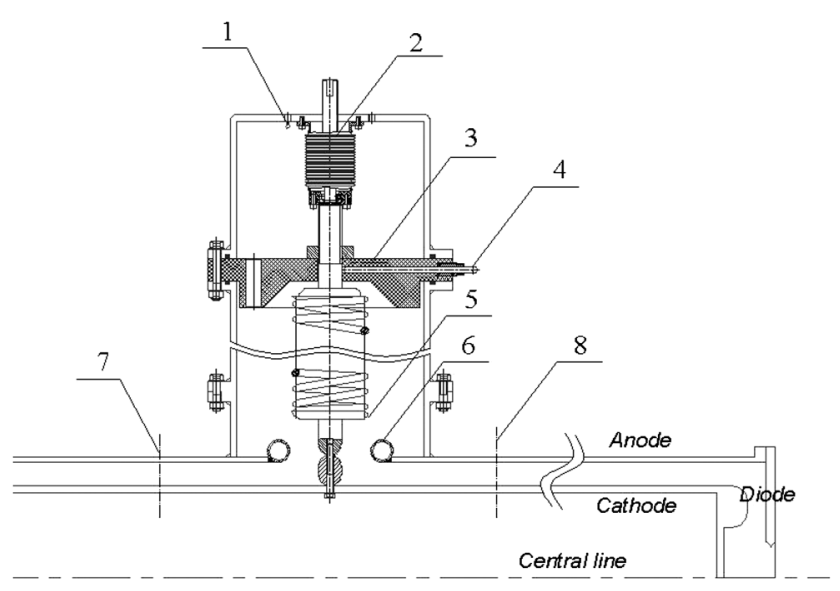

FIG. 6. Extensional MITL with high inductive helical support and diode load. B-dot (1) is used to record current of helical inductor. By changing the length of corrugated pipe (2), connecting and disconnecting of inductor (5) to ground are realized. A nylon insulation plate (3) helps to radially positioning the inductor. Grading structure (6) prevents electron drift into the inductor tube. Copper rod (4), inductor (5), and inner electrode of the MITL form current passage for premagnetizing pulse. Line (7) and line (8) denote cross sections where upstream and downstream current probes locate. 
simulation in Sec. IV shows the structure is effective to prevent electrons from entering the inductor tube.

\section{B. Extensional MITL design}

Theoretical investigations show that the operation point of a MITL is not unique at given voltage and vacuum impedance. Actually, there exists a working curve, which shows the anode current as a function of potential at the maximum extent of the flow pattern. The minimum current in the curve is defined as critical magnetic insulation current [13].

In our system, the transient time of MITL $(\sim 4 \mathrm{~ns})$ is negligible compared with pulse rise time. The MITL voltage and current is largely determined by load impedance, since voltage, current, and vacuum impedance determine the operation point of a MITL. Therefore, for a given load, vacuum impedance of the MITL dominates the establishing process of magnetic insulation and the pattern of electron flow. According to MITL theory, critical magnetic insulation current is inversely proportional to vacuum impedance. Therefore, different experiment states can be achieved by employing different extensional MITL and different load impedance.

Two extensional MITLs were used. The anode diameters were both $30 \mathrm{~cm}$. Difference existed in cathode dimensions. Diameters of 24 and $22 \mathrm{~cm}$ were used. Before experiment, we estimated their magnetic insulation characteristic and then roughly decided necessary conditions (charging voltage and diode impedance). Critical total current as a function of voltage was numerically calculated according to MITL theories of both Creedon [13] and Mendel [14,15]. The results are shown with curves in Fig. 7. Each curve divides the coordinate plane into two parts. Any operation point in the above part means its current is larger than the critical value and denotes a magnetic insulation state. Otherwise, magnetic insulation is not established.

Extensional MITL was terminated by a simple annular diode, whose A-K distance ranged from 15 to $35 \mathrm{~mm}$. A velvet cathode was used to form earlier and steadier electron emission. Figure 7 shows experimental states with different vacuum impedance, diode A-K distance, and charging voltage. Points with a hollow symbol denote magnetic insulation states, while the solid magenta pentagram denotes states without magnetic insulation. As mentioned above, voltage and current is load determined for our LTD system. Therefore, according to Fig. 7, with vacuum impedance of $18.6 \Omega$, magnetic insulation establishes easier than $13.4 \Omega$ for the same load.

\section{Probes}

B-dots were installed before and after the support inductor to measure the upstream and downstream current. In Fig. 6, lines (7) and (8) denote the cross sections

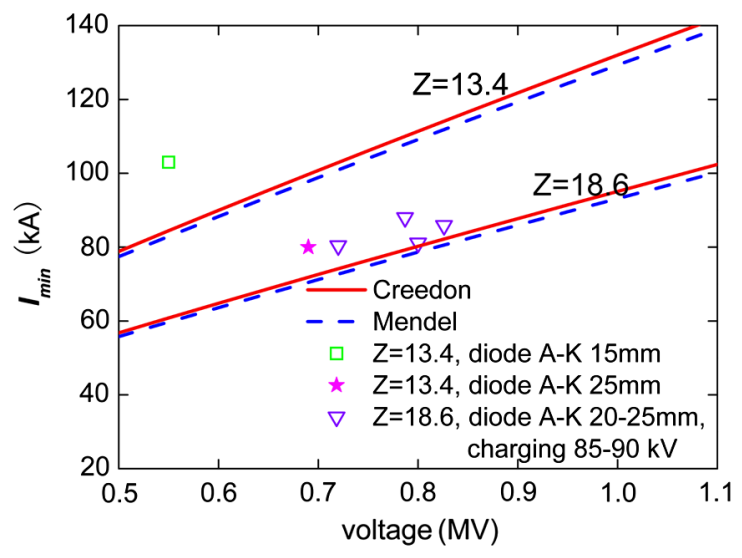

FIG. 7. $V-I_{\min }$ curves and discrete experimental states for the two line impedances. Red solid lines denote Creedon's theory, and blue dashed lines denote Mendel's theory. The hollow green square represents a magnetically insulated state with $Z=$ $13.4 \Omega$ and diode A-K of $15 \mathrm{~mm}$. The solid magenta pentagram represents an unmagnetically insulated state with $Z=13.4 \Omega$ and diode $\mathrm{A}-\mathrm{K}$ of $25 \mathrm{~mm}$. The purple triangles denote experimental state with $Z=13.4 \Omega$, diode A-K $20-25 \mathrm{~mm}$, and charging 85-90 kV. These states are magnetically insulated.

where upstream and downstream B-dots were placed. Four anode B-dots and two cathode B-dots were placed in each cross section. However, in experiments, only two anode B-dots and two cathode B-dots were used. Signals from them were rescaled and averaged to give current through the cross section. An extra B-dot [marked with (1) in Fig. 6] was used to record current of the helical support. By differentiating the current with time and multiply its sensitivity, MITL voltage was derived. A D-dot was installed on the MITL anode opposite the helical, serving as another voltage monitor. The waveform from this D-dot was used to judge magnetic insulation status.

All current B-dots were calibrated online at voltage lower than $100 \mathrm{kV}$ with a standard current probe which was installed at the load area, where a $10 \Omega \mathrm{KCl}$ water solution resistor was used instead of diode. No electron emission would happen at this voltage level. Therefore, cathode current, anode current, and load current have the same amplitude. A voltage probe was calibrated with a standard voltage probe at $15 \mathrm{kV}$. All calibrations were carried out if any part of the system was disassembled and reinstalled.

Calibration results suggest anode B-dots are much more stable than the cathode ones. Electron emission and influence of local electron flow might be the main factor to cause the difference. For anode B-dots, the standard deviations to their mean sensitivities are all below $3 \%$. Comparatively, $5 \%$ or even larger data exists for cathode B-dots. So following analysis to experimental results will mainly rely on anode current. 


\section{Experimental results with the inductive support}

Experiments with high inductive helical support were conducted for the two extensional MITLs (13.4 and $18.6 \Omega$ ) and different diode gaps, which was ranging from 15 to $35 \mathrm{~mm}$. Typical experimental states are plotted in Fig. 7 with scattered symbols. As suggested, not every state could establish magnetic insulation.

For the MITL with impedance of $13.4 \Omega$, the critical current is higher so that lower diode impedance is necessary to form magnetic insulation. In Fig. 7, the hollow green square point denotes an experiment state with $15 \mathrm{~mm}$ A-K distance for diode, whose impedance is about $5 \Omega$. For the state, the MITL voltage is about $550 \mathrm{kV}$. Anode currents before and after the helical inductor are 103 and $101 \mathrm{kA}$, and cathode currents are about 94 and $92 \mathrm{kA}$, respectively. Results indicate that electron current of the state is only a few $\mathrm{kA}$, which is believed to result from electron retrapping for undermatched load [16,17]. Since the current waveform oscillates seriously and damps slowly for such low impedance, the experiment state is harmful for both LTD and diode. Therefore, in other experiments, the diode with $15 \mathrm{~mm}$ or smaller A-K distance was not used any more. Also plotted in Fig. 7 with a solid pentagram is a state with A-K distance of $25 \mathrm{~mm}$ for the same MITL impedance. For the state, anode current is lower than critical value and magnetic insulation is not established. Evident current loss along the line happens.

In order to obtain sufficient useful results under harmless and magnetically insulated states with inductive support, a MITL impedance of $18.6 \Omega$ was used in the following experiments. Then magnetic insulation was effectively established when the diode gap is between 20 and $25 \mathrm{~mm}$. In these states, load impedance is about 8-10 $\Omega$. Accordingly, electron retrapping will be subordinate, and higher electron current is induced. Higher load impedance is also helpful to decrease the reverse pulse, which is beneficial to release operation risk and to prolong capacitor lifetime. Experiment states with MITL impedance of 18.6 $\Omega$ are plotted with a purple triangle in Fig. 7. For these states, a magnetic insulation characteristic could be judged with the help of $V-I_{\min }$ curves.

Figure 8 shows the anode currents for one of these states, in which the diode A-K distance is $21 \mathrm{~mm}$ and LTD capacitors are charged to $85 \mathrm{kV}$. The red solid line and black dashed line denote current before and after the helical inductor, which were derived and averaged from two anode B-dots in cross sections [lines (7) and (8) in Fig. 6] perpendicular to the power flow direction. The amplitudes are 82.6 and $80.4 \mathrm{kA}$, respectively, suggesting about $2 \mathrm{kA}$ loss across the helical support. Voltage for this state is about $720 \mathrm{kV}$. Therefore, the diode impedance is nearly $9 \Omega$. According to numerical calculation, the critical current corresponding to the state is about $73 \mathrm{kA}$. So both upstream and downstream of the line are magnetically insulated.

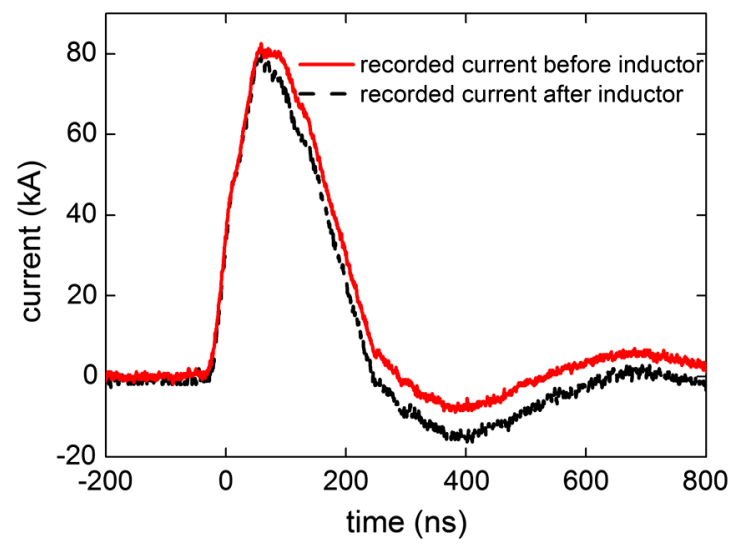

FIG. 8. Recorded anode current before and after the helical inductor with line impedance of $18.6 \Omega$. Each of the current waveforms is averaged from two B-dot signals in the same cross section perpendicular to the power flow direction. The maximum currents before and after the inductor are 82.6 and $80.4 \mathrm{kA}$. The MITL voltage is about $720 \mathrm{kV}$.

Figure 8 also clearly shows the difference of the two waveforms at their trailing edges. The fact indicates that current shunting from the inductor is the main reason resulting in the current loss. This is because, for the inductor, current rises slower than the major loop and the influence to total current becomes evident at the trailing edge of the pulse. In other words, if electron loss is evident and should be responsible for the total current loss around the inductor, the rising edges of the two current waveforms will be different from each other.

\section{E. Experimental results without the inductive support}

A nonsupport setup was realized by replacing the tee joint with a straight tube and removing the helical in Fig. 6 .

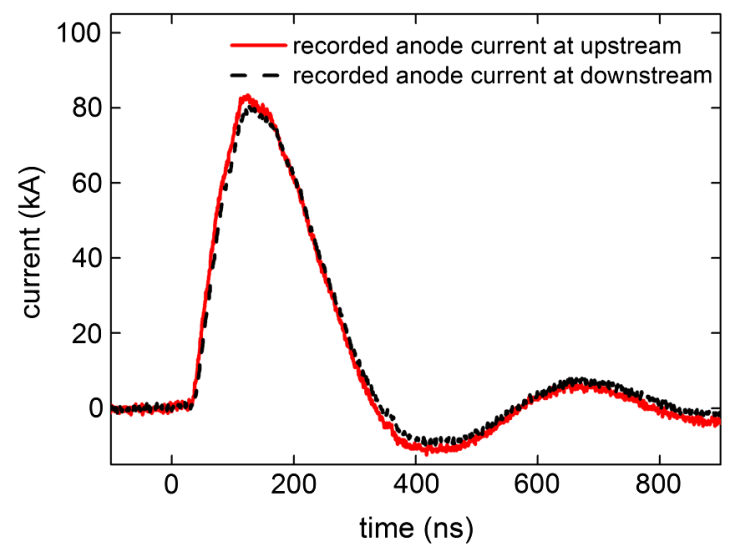

FIG. 9. Upstream and downstream anode currents in nonsupport experiment. Currents are recorded at the same positions and postprocessed in the same manner as the support experiment. The maximum current is $83.4 \mathrm{kA}$ at upstream and is $81.1 \mathrm{kA}$ at downstream. Waveforms overlap well at both rising and trailing edges. 
With approximately identical conditions with the support experiment (the diode A-K distance might have about $1 \mathrm{~mm}$ difference due to assembling operation), upstream and downstream anode current were recorded.

As shown in Fig. 9, the current waveforms in the nonsupport experiment agree very well in both rise and trailing edges. However, the amplitudes have a difference of about $2 \mathrm{kA}$ ( $83.4 \mathrm{vs} 81.1 \mathrm{kA})$. One possible reason resulting in the difference might be uncertainty in scaling coefficient for B-dots. Another possibility is that there is real current loss between the two diagnostic positions for the state due to insufficient magnetic insulation. This is because in this experiment, the MITL voltage is a bit higher than the support situation (removing the helical support away actually increases the equivalent impedance of load, which partially results in higher working voltage for MITL), which means higher critical current is needed. Therefore, the nonsupport experiment state might be very critical for magnetic insulation.

\section{PARTICLE-IN-CELL SIMULATIONS}

PIC simulations were conducted with CHIPIC [18], a 3D paralleled code. The purpose of the simulations is to verify the electron flow behavior when it passes the support inductor, and to study how much the effect to the magnetic insulation will be. Power flow in the LTD system is axial symmetrical and can be characterized with a twodimensional (2D) model if there is no helical support. With the helical support, three-dimensional (3D) simulation is necessary to study the influence of helical support to MITL power flow.

The Cartesian system is used to facilitate the modeling to the helical structure. The $Z$ direction is defined as the power flow direction. The simulation configuration is the same as that of Fig. 6, in which the $1.2 \mathrm{~m}$ extensional MITL and a diode are included. The total length of simulation area is about $1.3 \mathrm{~m}$. Anode and cathode diameters are 30 and $22 \mathrm{~cm}$, respectively. The helical inductor locates at $z=$ $0.496 \mathrm{~m}$. The tube surrounding the inductor has a diameter of $24.5 \mathrm{~cm}$. According to a cold test by CHIPIC, the equivalent inductance of the helical setup is about $9.8 \mu \mathrm{H}$. The length of the inductor is $0.3 \mathrm{~m}$ with 19.5 windings. The diameter of winding is $10 \mathrm{~cm}$. The bottom end of the helical is connected to the MITL cathode with a $12 \mathrm{~cm}$ long straight rod, whose diameter is $3 \mathrm{~cm}$. All these simulations have the same parameters compared with the experiments.

The connection to the top end of the inductor is simplified. A $17 \mathrm{~cm}$ long rod (diameter of $3 \mathrm{~cm}$ ) is used to replace the complex setups in the real situation. According to experiments, we believe that electron emission was not turned on for the helical. Therefore, in simulation, although specified as conductor, electron emission is not allowed from the helical and the two rod surfaces.

The load in the simulation is an annular diode. The width of the cathode ring is $55 \mathrm{~mm}$. The A-K distance is set to
$22 \mathrm{~mm}$. Explosive electron emissions are initiated from the cathode as the field exceeds $20 \mathrm{kV} / \mathrm{cm}$, while the electron emission threshold for the MITL cathode is $200 \mathrm{kV} / \mathrm{cm}$. The thresholds correspond to aluminum for MITL and velvet for diode.

Equivalent impedance of the inductance depends on the temporal frequency of the driving pulse, so the shape of the voltage pulse driving the simulation configuration from the left port is derived from experiments. In order to make quantitative comparison, we changed the voltage amplitude around 1 MV (the nominal forward wave of the system with matched load) with a trial and error method until the measured MITL voltage at the helical is about $720 \mathrm{kV}$, which is the value recorded by the voltage probe in experiment.

A uniform grid is used in the $X$ direction with a size of $2.5 \mathrm{~mm}$. In $Y$ and $Z$ directions, grid size is $5 \mathrm{~mm}$ except for the helical and diode areas. The simulation is conducted on a Windows-based platform, whose processor is Intel Core i5-2400 and memory is 4 GB. Typically, about 37.6 hours is needed to finish one single simulation to $180 \mathrm{~ns}$.

Voltage and current at different $Z$ positions were diagnosed in simulations. Figure 10 shows simulated MITL voltage at $z=0.496 \mathrm{~m}$, where the helical was placed. The black line represents the original signal by integrating the absolute electric field across the A-K gap. The red line, whose maximum is $718 \mathrm{kV}$, denotes average smoothing to adjacent points with $1 \mathrm{~ns}$ constant. The drop and re-rise near

$t=50 \mathrm{~ns}$ implies the beginning of electron emission.

Figures 11-13 illustrate simulated anode currents, cathode currents, and electron currents before $(z=0.3 \mathrm{~m})$ and $\operatorname{after}(z=0.692 \mathrm{~m})$ the helical inductor. Especially, anode current close to diode $(z=1.22 \mathrm{~m})$ is also plotted to examine the influence of helical to further area. It can be seen from Fig. 11 that the simulated maximum anode

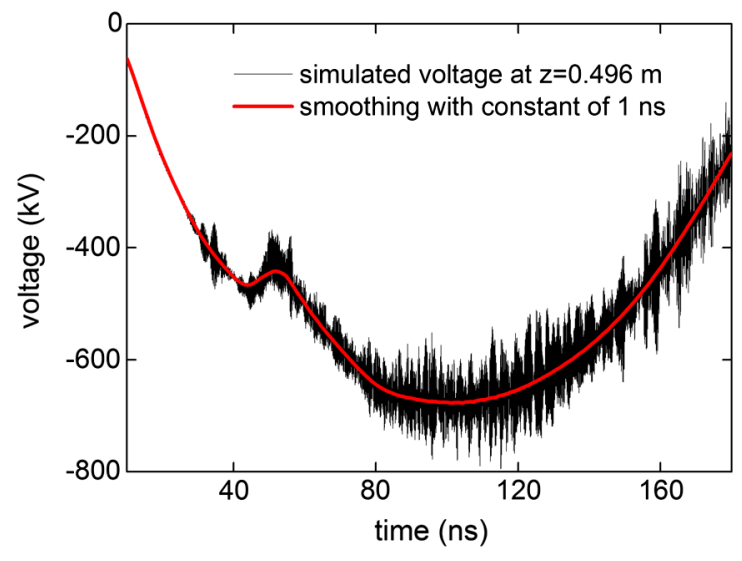

FIG. 10. Simulated MITL voltage at $z=0.496 \mathrm{~m}$, where helical support was placed. The black line represents signal by integrating the absolute electric field across the A-K gap. The red line denotes average smoothing to adjacent points with $1 \mathrm{~ns}$ constant. The maximum of the red line is $718 \mathrm{kV}$. 


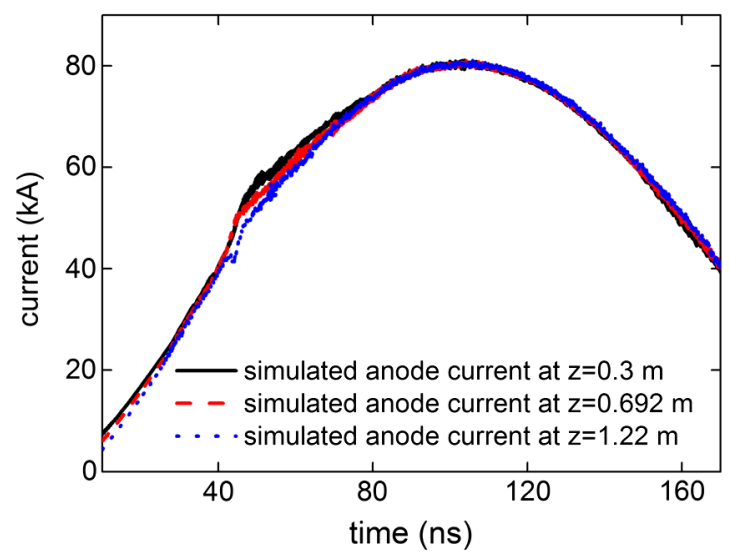

FIG. 11. Simulated anode currents at $z=0.3 \mathrm{~m}, z=0.692 \mathrm{~m}$, and $z=1.22 \mathrm{~m}$, corresponding to position before and after the helical support $(z=0.496 \mathrm{~m})$ and a position vicinity to diode. The amplitudes are $81.6,81.1$, and $81.0 \mathrm{kA}$, respectively, suggesting very small loss along the line.

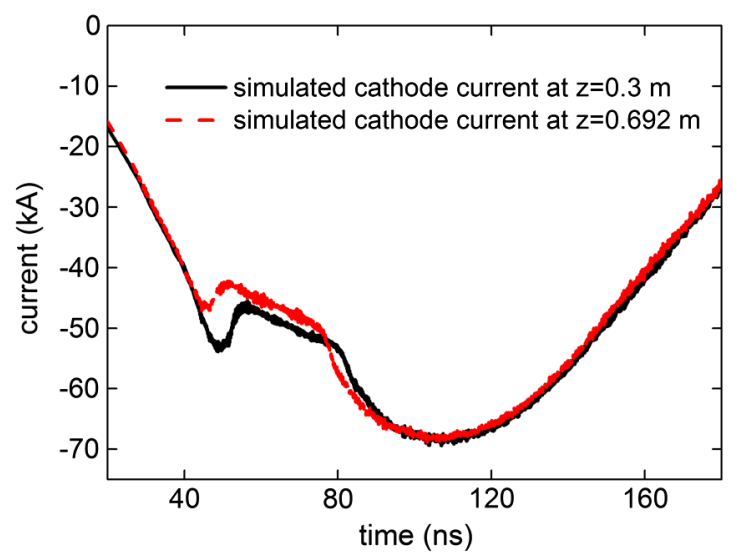

FIG. 12. Simulated cathode currents at $z=0.3 \mathrm{~m}$ and $z=$ $0.692 \mathrm{~m}$. Inferring from the curves, electron emission starts earlier at downstream of the support, which is believed to be resulted from field enhancement at the contact area of helical and MITL cathode. Inflexion around 70 ns suggests effective magnetic insulation starts to establish.

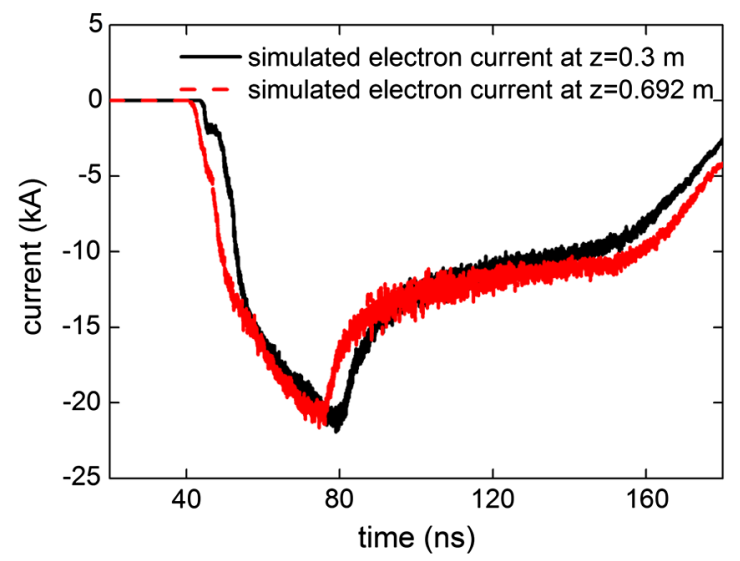

FIG. 13. Simulated electron currents at $z=0.3 \mathrm{~m}$ and $z=$ $0.692 \mathrm{~m}$. It can be inferred that electron emission turns on earlier at downstream, but the employment of support does not result in extra electrons.

currents are about $81 \mathrm{kA}$, which is very close to the value recorded in experiment. Figures 12 and 13 show that electron emission is turned on earlier at the downstream area. This is due to electric field enhancement at the contact area of the helical and MITL cathode. According to Figs. 12 and 13, cathode current and electron current increase before $t=80 \mathrm{~ns}$. After that, magnetic insulation is effectively established.

Inferring from the waveforms, the upstream and downstream of MITL show very similar behaviors. The influence of helical support to power flow is limited, except for the beginning of electron emission. No evident current loss exists between $z=0.3 \mathrm{~m}$ and $z=1.22 \mathrm{~m}$. Even at the trailing edge, the three anode current waveforms nearly overlap with each other.

Figure 14 shows electron phase space in the $Y Z$ plane at two different moments. Figures 14(a) and 14(b) show phase space at $t=71 \mathrm{~ns}$ and $t=108 \mathrm{~ns}$, respectively. They correspond to the moments at rise edge and near the maximum current. As can be seen from Fig. 14, the existence of the inductor makes electron flow partially distorted, but the influence is local and limited. In the short
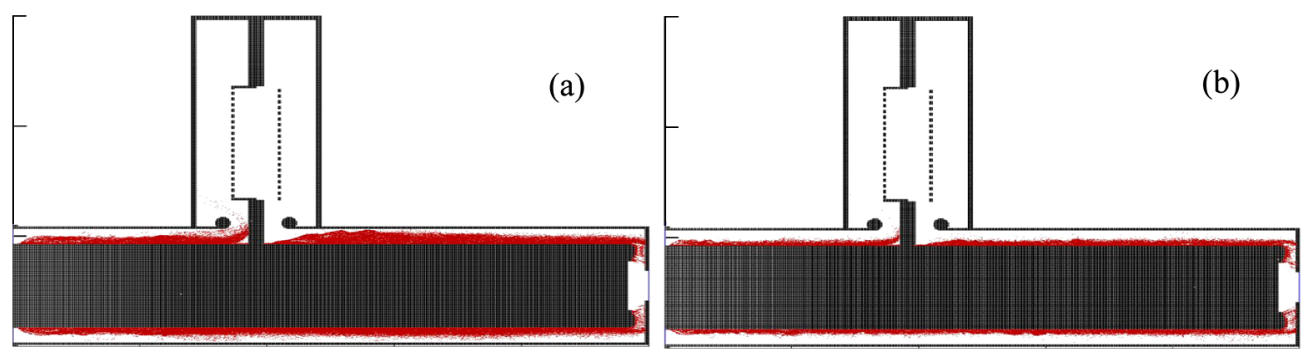

FIG. 14. Electron phase space in the $Y Z$ plane at different moments. Part (a) represents $t=71 \mathrm{ns,}$ a moment close to inflexion of curves in Fig. 12. As can be seen, although a fraction of electrons strike onto the support rod, electrons are insulated from anode nearly everywhere. Part (b) represents $t=108 \mathrm{~ns}$, at which current reaches almost the maximum, magnetic insulation is established very well. 
distance at downstream, magnetic insulation recovers soon. That is why slight differences exist between the three curves in Fig. 11.

Figure 15 shows an electron phase space matrix in the $X Y$ plane. Axes are not shown in the figures. The $X$ axis lays in the horizontal and $Y$ in the vertical. For a single phase space, the outer circle represents anode, and the inner circle represents cathode. Facing the A-K gaps formed by two circles, the projection of helical support is at the top. At the moment of $t=71 \mathrm{~ns}$, although covering near half of the A-K gap, electrons cannot reach anode any more. At $z=0.692 \mathrm{~m}$, which is $20 \mathrm{~cm}$ downstream of the helical support, electron flow goes higher at the top of the A-K gap. However, at $z=1.22 \mathrm{~m}$, electron flow becomes nearly the same with that of $z=0.3 \mathrm{~m}$. At $t=108 \mathrm{~ns}$, when current is reaching the maximum, magnetic insulation is established very well. The phase space for the three different positions is almost the same.

According to these simulations, we believe that the existence of high inductive helical support in the LTD system has very slight influence on MITL power flow. Meanwhile, the approximately equal current in experiment and simulation prove the reliability of probes.

We also conducted simulations with electron emission on the helical surface to exam the influence to helical performance. The simulations were conducted under different conditions with that of Fig. 14. Cathode diameter is $24 \mathrm{~cm}$ (vacuum impedance $13.4 \Omega$ ). MITL voltage is $550 \mathrm{kV}$. A-K distance of the diode is $15 \mathrm{~mm}$. In that case, plenty of electrons are pulled out from the helical surface (see Fig. 16) and evident current loss occurs. This means, first, the judgment that no electron emission on helical surface in our experiment is reasonable, and second, the employment of high inductive helical support

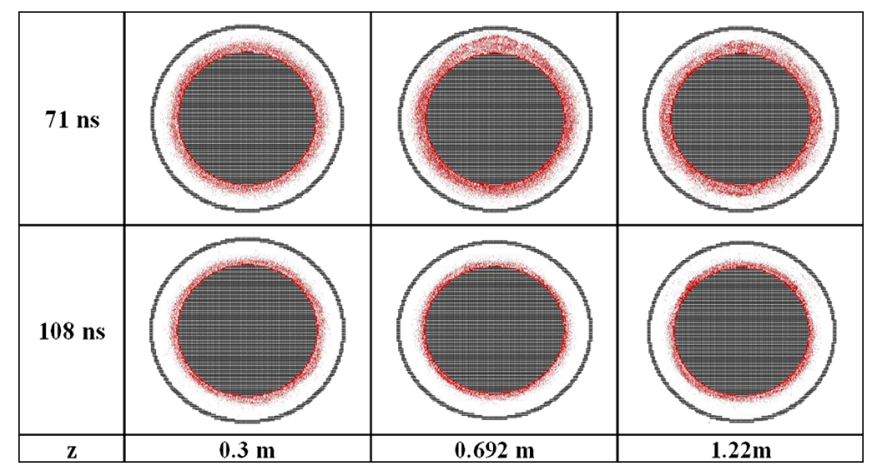

FIG. 15. Electron phase space matrix in the $X Y$ plane at different positions. The $X$ axis lays in the horizontal, and $Y$ axis in the vertical. The outer circles represent anodes, and inner circles represent cathodes. The helical support is at the top of the A-K gap and $z=0.496 \mathrm{~m}$. As can be seen, even at the rise edge of current $(t=71 \mathrm{~ns})$, the influence of support to electron flow is weak and electron flow can recover in a short distance. At the moment close to maximum $(t=108 \mathrm{~ns})$, phase space for the three different positions is almost the same.

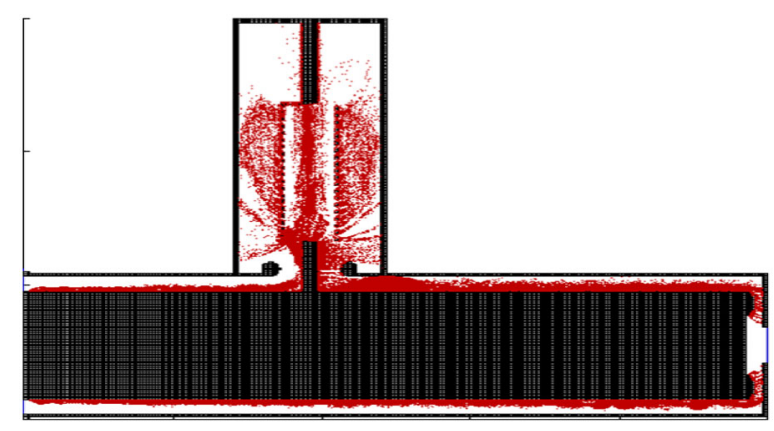

FIG. 16. Electron phase space in the $Y Z$ plane with electron emission on the helical surface. This simulation was conducted under different conditions with that of Fig. 14 to exam the influence of electron emission to helical performance. The cathode diameter is $24 \mathrm{~cm}$ and MITL voltage is $550 \mathrm{kV}$. The A-K distance of the diode is $15 \mathrm{~mm}$. Electron emission turns on when the electric field exceeds $20 \mathrm{kV} / \mathrm{cm}$ for helical. The simulated anode currents are $113 \mathrm{kA}$ at $z=0.3 \mathrm{~m}$ and $103.5 \mathrm{kA}$ at $z=0.692 \mathrm{~m}$, indicating nearly $10 \mathrm{kA}$ loss across the helical support.

with voltage up to several megavolts might be impossible, where the electric field would exceed the electron emission threshold and arc will be induced between windings. Actually, electron emission will greatly reduce the effective impedance of the helical and lead to serious current loss. Therefore, we believe that preventing electron emission from the helical surface is very fundamental to use it as support for MITL. In principle, two measurements are worth considering. The first one is to use material with very high emission threshold, and the other is to lengthen windings distance to make the field strength lower than the emission threshold. But they are not easy in engineering for rather high voltage.

\section{SUMMARY AND CONCLUSIONS}

In this paper, we have demonstrated by experiments on a 10-stage LTD system and by 3D PIC simulations that high inductive helical can be used to serve as support to an electrode in the MITL as well as entry of premagnetizing current simultaneously. The experiment and simulation results are consistent with each other. However, there are still some differences. The efficiency of the support structure in the simulation is higher than experiments, but more current loss is recorded in experiment. Regarding the current waveforms, experimental results has shown a difference in the trailing edge for currents before and after the support. But in the simulations, currents before and after the support are almost the same. The reason for this difference needs further investigation.

According to the investigations, we believe that the employment of high inductive helical as a support of MITL influences the power flow slightly in our LTD system. Although electron flow is locally altered, magnetic insulation recovers soon at downstream. At voltage close 
to our LTD system, we conclude that a high inductive structure can be used to support the MITL cathode. However, when scaling the technique to a voltage of several megavolts, there exist practical limitations. The most fatal question is electron emission on the surface of the support. According to PIC simulations, we find serious loss will be induced if electron emission happens on the support. In order to avoid electron emission, longer winding distance and helical length are needed. According to Ref. [12], the helical inductance increases slowly with its length. Therefore, at a voltage as high as several megavolts, the length of the helical has to be a large value to ensure the surface electric field below emission threshold. The second one is the strength of the helical. For a long helical, it is difficult to make it stiff enough to constrain the length change within a reasonable value. A large deformation will result in serious deviation in effective impedance, which will result in considerable current loss.

\section{ACKNOWLEDGMENTS}

The authors would like to thank Jing Ren, Bo Jin, Jie Wang, Yuanjun Zhang, and Ye Li for experimental operation and pulsed-power system maintaining.

[1] M. S. Di Capua , IEEE Trans. Plasma Sci. 11, 205 (1983).

[2] W. A. Stygar, P. A. Corcoran, H. C. Ives, R. B. Spielman, J. W. Douglas, B. A. Whitney, M. A. Mostrom, T. C. Wagoner, C.S. Speas, T.L. Gilliland, G. A. Allshouse, R. E. Clark, G. L. Donovan, T.P. Hughes, D. R. Humphreys, D. M. Jaramillo, M. F. Johnson, J. W. Kellogg, R. J. Leeper, F. W. Long, T. H. Martin, T. D. Mulville, M. D. Pelock, B. P. Peyton, J. W. Poukey, J. J. Ramirez, P. G. Reynolds, J. F. Seamen, D. B. Seidel, A. P. Seth, A. W. Sharpe, R. W. Shoup, J. W. Smith, D. M. Van De Valde, and R. W. Wavrik, Phys. Rev. ST Accel. Beams 12, 120401 (2009).

[3] Feng Shuping, Li Hongtao, Xie Weiping, Deng Jianjun, Xia Minghe, Ji Ce, Wang Meng, Guan Yongchao, and He An, High Power Laser Part. Beams 21, 463 (2009).

[4] W. A. Stygar, M. E. Cuneo, D. I. Headley, H. C. Ives, R. J. Leeper, M. G. Mazarakis, C. L. Olson, J. L. Porter, T. C. Wagoner, and J.R. Woodworth, Phys. Rev. ST Accel. Beams 10, 030401 (2007).

[5] J. J. Ramirez, K. R. Prestwich, E. L. Burgess, J. P. Furaus, R. A. Hamil, D. L. Johnson, T.W. L. Sanford, L. O. Seamons, L.X. Schneider, and G.A. Zawadzkas, in
Proceedings of the 6th IEEE International Pulsed Power Conference (1987), p. 294.

[6] David L Johnson, Robert Altes, Vernon Bailey, Patrick Corcoran, Ian Smith, Steve Cordova, Fawn Griffin, Kelly Hahn, Deanna Jaramillo, John Maenchen, Isidro Molina, Salvadore Portillo, Elizabeth Puetz, Matt Sceiford, David Van De Valde, David Rose, Bryan Oliver, Dale Welch, Dennis Barker, Darryl Droemer, Ray Gignac, Frank Wilkins, and Bradley Shelton, in Proceedings of the 13th International Symposium on High Current Electronics, Tomsk (2004), p. 115.

[7] E. L. Burgess, G.W. Crowder, M.C. Dowdican, J.C. Patterson, T. L. Franklin, S. J. Robischon, K. M. Tolk, J. J. Ramirez, D. L. Johnson, and R.C. Pate, in Proceedings of the 6th IEEE International Pulsed Power Conference (1987), p. 506.

[8] A. A. Kim, B. M. Kovalchuk, A. N. Bastrikov, V. G. Durakov, S. N. Volkov, and V.A. Sinebryukhov, in Proceedings of the 12th IEEE International Pulsed Power Conference (2001), p. 1491.

[9] A. A. Kim, M. G. Mazarakis, V. A. Sinebryukhov, B. M. Kovalchuk, V.A. Visir, S. N. Volkov, F. Bayol, A. N. Bastrikov, V. G. Durakov, S. V. Frolov, V. M. Alexeenko, D. H. McDaniel, W. E. Fowler, K. LeChien, C. Olson, W. A. Stygar, K. W. Struve, J. Porter, and R. M. Gilgenbach, Phys. Rev. ST Accel. Beams 12, 050402 (2009).

[10] Joshua J. Leckbee, John E. Maenchen, David L. Johnson, Salvador Portillo, David M. VanDeValde, David V. Rose, and Bryan V. Oliver, IEEE Trans. Plasma Sci. 34, 5 (2006).

[11] L. Chen, L. Zhou, W. Zou, J. Ren, Y. Li, S. Wu, W. Xie, S. Feng, J. Deng, Acta Phys. Pol. A 115, 6 (2009).

[12] P. L. Kalantarov and L. A. Tseitlin, Inductance Calculation Manuals (The Atomic Energy Press, 1986), translated into Chinese by Chen Tangmin and Lin Bao'an, China Machine Press, Beijing, 1992.

[13] J. M. Creedon, J. Appl. Phys. 48, 3 (1977), p. 1070.

[14] C. W. Mendel, Jr. and S. E. Rosenthal, Phys. Plasmas 2, 1332 (1995).

[15] P.F. Ottinger and J. W. Schumer, Phys. Plasmas 13, 063109 (2006).

[16] S. Ya. Belomittsev, A. A. Kim, A. V. Kirikov, and V. V. Ryzhov, in Proceedings of the 14th International Symposium on High Current Electronics (2006), p. 236-239.

[17] Zou Wenkang, Deng Jianjun, and Song Shengyi, High Power Laser Part. Beams 19, 993 (2007).

[18] CHIPIC is a paralleled 3D particle-in-cell code by University of Electronic Science and Technology of China. 